\title{
PDCD2 sensitizes HepG2 cells to sorafenib by suppressing epithelial-mesenchymal transition
}

\author{
HONGYU LIU ${ }^{1}$, MIN WANG ${ }^{2}$, NA LIANG $^{3}$ and LIANYUE GUAN ${ }^{1}$ \\ ${ }^{1}$ Department of Hepatobiliary-Pancreatic Surgery, China-Japan Union Hospital of Jilin University, \\ Changchun, Jilin 130033; ${ }^{2}$ Department of Pathology, Jilin Cancer Hospital, Changchun, Jilin 130012; \\ ${ }^{3}$ Office of Surgical Nursing, Changchun Medical College, Changchun, Jilin 130000, P.R. China
}

Received April 12, 2018; Accepted November 5, 2018

DOI: $10.3892 / \mathrm{mmr} .2019 .9860$

\begin{abstract}
Epithelial-mesenchymal transition (EMT) has an established role in the acquisition of therapeutic resistance. Programmed cell death domain 2 (PDCD2) is involved in the progression of multiple types of cancer. However, its mechanism underlying chemoresistance in liver cancer has not been elucidated. In the present study, it was demonstrated that the sorafenib-resistant HepG2 cell line exhibited EMT and multidrug resistance (MDR) phenotypes, and reduced expression of PDCD2, by reverse transcription-quantitative polymerase chain reaction (RT-qPCR), western blot analysis and Cell Counting Kit-8. Annexin V/fluorescein isothiocyanate and cell migration assays further demonstrated that PDCD2 effectively promoted sorafenib-induced cell apoptosis and reduced cell metastasis. Mechanistically, PDCD2 inhibited the expression of Vimentin and increased the expression of E-cadherin in a Snail-dependent manner by RT-qPCR and western blot analysis. In conclusion, the present study elucidated for the first time, to the best of our knowledge, that PDCD2 sensitizes sorafenib-resistant HepG2 cells to sorafenib by the downregulation of EMT. PDCD2 may serve as a potential therapeutic target in the treatment of sorafenib-resistant liver cancer.
\end{abstract}

\section{Introduction}

Programmed cell death domain 2 (PDCD2), is a highly conserved zinc finger MYND domain-containing protein and is expressed in a variety of tissues (1). The original PDCD2 clone (RP-8) was isolated from a rat gene that was

Correspondence to: Dr Lianyue Guan, Department of Hepatobiliary-Pancreatic Surgery, China-Japan Union Hospital of Jilin University, 126 Xiantan Street, Changchun, Jilin 130033, P.R. China

E-mail: 13504310464@163.com

Abbreviations: EMT, epithelial-mesenchymal transition; MDR, multidrug resistance; PDCD2, programmed cell death domain 2

Key words: PDCD2, sorafenib, EMT, Snail associated with programmed cell death (2). Generally, PDCD2 contributes to stem cell activity and tissue remodeling by the induction of apoptosis (3). Accumulating data demonstrated that PDCD2 is involved in the development of cancer. For example, the expression of PDCD2 is decreased in gastric cancer tissue, and it may induce gastric cancer cell growth arrest and apoptosis in a p53-dependent manner (4,5). PDCD2 serves as a tumor suppresser gene involved in the pathogenesis of osteosarcoma (3). However, its functions in carcinogenesis are debatable. For example, in human acute leukemia cells, PDCD2 was identified to be expressed at a high level, and its knockdown impaired cancer cell proliferation, suggesting that PDCD2 significantly facilitates leukemia progression (6). A previous study demonstrated that PDCD2 is downregulated in drug-resistant breast cancer cells, indicating that PDCD2 may be involved in the process of the acquisition of multidrug resistance (MDR) (7). However, at present, the underlying mechanism of the involvement of PDCD2 in drug resistance in liver cancer cells remains to be elucidated.

Liver cancer is the fifth most common type of cancer worldwide, and is the third most frequent cause of cancer-associated mortality to the poor prognosis and rapid progression (8). Chemotherapy remains an optional treatment for liver cancer. However, drug resistance in patients diagnosed with liver cancer frequently leads to the failure of chemotherapeutic administration (9). At present, the molecular mechanisms underlying drug resistance remain to be fully understood. Elucidating the molecular mechanisms of MDR is urgently required for the development of effective chemotherapeutic drugs. The activation of epithelial-mesenchymal transition (EMT) serves a principal role in the process of MDR (10). Cancer stem cell (CSC)-like cells may facilitate tumor cell acquisition of chemotherapy and radiotherapy resistance by the activation of EMT (11). The CSC-like cells are responsible for drug resistance and tumor metastasis, and are the principal reason for tumor treatment failure and cancer-associated mortality (12). Clinically, sorafenib is the first-line treatment drug to prolong the overall survival rate of patients with advanced liver cancer (13). However, drug resistance of sorafenib remains a primary challenge in improving the prognoses of patients with liver cancer (14). Generally, sorafenib exerts an inhibitory function against EMT via the inhibition of mitogen-activated protein kinase (MAPK) signaling and expression of Snail in 
liver cancer (15). However, sorafenib-resistant liver cancer cells exhibit EMT and MDR phenotypes, indicating that EMT is important in sorafenib-resistant liver cancer cells $(16,17)$. Therefore, identifying the molecular mechanism underlying sorafenib resistance is indispensable for the development of effective chemotherapeutic treatments.

In the present study, it was demonstrated that PDCD2 was decreased in the sorafenib-resistant HepG2 cell line and that the overexpression of PDCD2 increased the sensitivity of chemoresistant HepG2 cells to sorafenib. Following experiments demonstrated that PDCD2 increased the expression of apoptotic proteins, suppressed resistant HepG2 cell metastasis and led to an elevated apoptotic rate when treated with sorafenib. Mechanistically, PDCD2 inhibited EMT, possibly in a Snail-dependent manner. Taken together, the present study preliminarily demonstrated that PDCD2 serves as a pivotal molecule to overcome therapy failure in the treatment of resistant liver cancer.

\section{Materials and methods}

Cell line and vectors. The HepG2 human liver cancer cell line was obtained from the Shanghai Institute of Cell Biology, Chinese Academy of Sciences (Shanghai, China). The related sorafenib-resistant cell line (HepG2/SF) was generated by exposing cells to increasing concentrations $(\leq 2 \mu \mathrm{M})$ of sorafenib. The MDR phenotype was evidenced by the half maximal inhibitory concentration (IC50; data not shown). These cells were maintained in RPMI-1640 (Gibco; Thermo Fisher Scientific, Inc., Waltham, MA, USA) supplemented with $10 \%$ fetal bovine serum (FBS, Gibco; Thermo Fisher Scientific, Inc.) at $37^{\circ} \mathrm{C}$ in a $5 \% \mathrm{CO}_{2}$ humidified incubator. The PDCD2 expression vector was constructed in the Laboratory of Hepatobiliary-Pancreatic Surgery (Jilin University, Jilin, China). The PDCD2 lentiviral vector and Snail interfering vector (Snail-sh) were constructed by Shanghai GeneChem Co., Ltd. (Shanghai, China), and were transfected into HepG2 cells at a 10 multiplicity of infection (MOI) value. After $48 \mathrm{~h}$, the cells were used in subsequent experiments.

Analysis of cell viability. Cell viability was detected using the Cell Counting Kit-8 (Dojindo Molecular Technologies, Inc., Shanghai, China). Each experiment was repeated three times. In detail, the sorafenib-resistant cell line (HepG2/SF) was plated into 96 -well plates with a total of $2 \times 10^{3}$ cells/well and subsequently treated with sorafenib (concentrations ranged between 0 and $15 \mu \mathrm{M}$ ) at $37^{\circ} \mathrm{C}$ for $24 \mathrm{~h}$ following transfection with or without PDCD2. The cytotoxicity index was calculated as (1-OD450 of drug-treated cells/OD450 of untreated cells) x100 according to a previous study (18). The IC50 values of sorafenib were calculated using GraphPad Prism 5 (GraphPad Software, Inc., La Jolla, CA, USA).

Transfection and cell migration assay. PDCD2 lentiviral vector was transfected into HepG2 cells at a $10 \mathrm{MOI}$ value, according to the manufacturer's protocol (Shanghai GeneChem Co., Ltd.). After $48 \mathrm{~h}$, the cells were assessed to detect cell migration using polycarbonate membranes with an $8-\mu \mathrm{m}$ pore size (Corning, Inc., Corning, NY, USA). The cells $\left(6 \times 10^{4}\right)$ were seeded into the upper chamber with $200 \mu$ l serum-free medium, and the upper chambers were subsequently placed onto the lower chambers of 24-well culture dishes containing $500 \mu \mathrm{l}$ RPMI-1640 containing 10\% FBS. After $48 \mathrm{~h}$, cells that had migrated to the outer side of the membranes were fixed with $4 \%$ paraformaldehyde for $30 \mathrm{~min}$ at room temperature and stained with $0.1 \%$ crystal violet for $20 \mathrm{~min}$ at room temperature. The number of migrated cells was counted under a light microscope (magnification, x100; Olympus CKX31; Olympus Corporation, Tokyo, Japan). Consistently, the snail interfering vector (Snail-sh) was additionally transfected into HepG2 cells at a 10 MOI value, and after $48 \mathrm{~h}$, the cells were used in the following experiments.

Western blot analysis. The total proteins from cells were extracted and prepared using radioimmunoprecipitation assay buffer containing phenylmethanesulfonyl fluoride (Thermo Fisher Scientific, Inc.). Lysates were subsequently centrifuged at $11,000 \mathrm{x}$ g for $15 \mathrm{~min}$ at $4^{\circ} \mathrm{C}$. The protein concentration was determined using a Bicinchoninic Acid Protein Assay kit (Thermo Fisher Scientific, Inc., Waltham, MA, USA). A total of $15 \mu \mathrm{g}$ proteins in the supernatant were separated on $10 \%$ SDS-PAGE and subsequently transferred to polyvinylidene difluoride membranes. TBS with Tween 20 containing $5 \%$ nonfat milk powder (w/v) was used to block the membranes for $2 \mathrm{~h}$ at room temperature. The membranes were incubated with primary antibodies against PDCD2 (1:2,000; cat. no. ab133324; Abcam, Cambridge, MA, USA), MDR1 (1:1,000; cat. no. 901401; BioLegend, Inc., San Diego, CA, USA), matrix metalloproteinase (MMP)2 (1:2,000; cat. no. 87809; Cell Signaling Technology, Inc., Danvers, MA, USA), MMP9 (1:2,000; cat. no. 2270; Cell Signaling Technology, Inc.), Caspase 3 (1:1,000; cat. no. 9665; Cell Signaling Technology, Inc.), Vimentin (1:1,000; cat. no. 5741; Cell Signaling Technology, Inc.), E-cadherin (1:1,000; cat. no. 3195; Cell Signaling Technology, Inc.) and Snail (1:1,000; cat. no. 3895; Cell Signaling Technology, Inc.) for $12 \mathrm{~h}$ at $4^{\circ} \mathrm{C}$, respectively. GAPDH was used as the internal control (1:1,000; cat. no. 5174; Cell Signaling Technology, Inc.). The membranes were subsequently incubated with horseradish peroxidase-conjugated secondary antibodies (1:5,000; cat. nos. A27022 and A16169; Thermo Fisher Scientific, Inc.) for $2 \mathrm{~h}$ at room temperature. An Enhanced Chemiluminescent Substrate Reagent kit (Thermo Fisher Scientific, Inc.) was used to detect the bound antibodies. Finally, protein expression was quantified using the Carestream IS4000MM Pro Molecular Imaging System (cat. no. 8642985; Carestream Health, Inc., Rochester, NY, USA) and analyzed with ImageJ software (version no. 1.4.3.67; National Institutes of Health, Bethesda, MD, USA). All experiments were performed in triplicate independently.

Evaluation of apoptosis. The cells were transfected with PDCD2 and were subsequently treated with sorafenib for $24 \mathrm{~h}$. The apoptotic cells were assessed using an Annexin V/fluorescein isothiocyanate and propidium iodide apoptosis detection kit (Dojindo Molecular Technologies, Inc.). Flow cytometry was used to measure apoptosis using a Beckman Coulter flow cytometer (Beckman Coulter, Inc., Brea, CA, USA) and the data were analyzed with Kaluza software (version. no 2.0; Beckman Coulter, Inc.). 
A

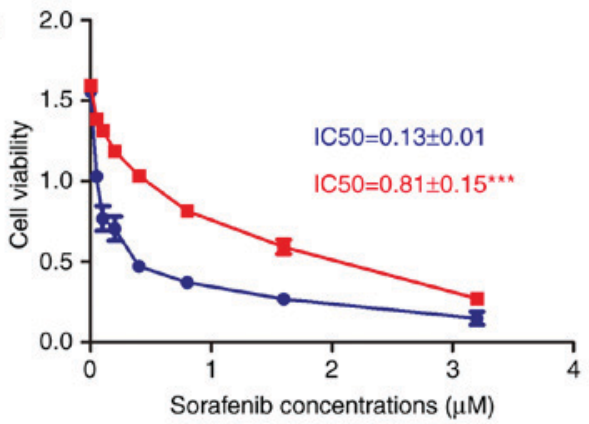

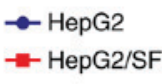

B

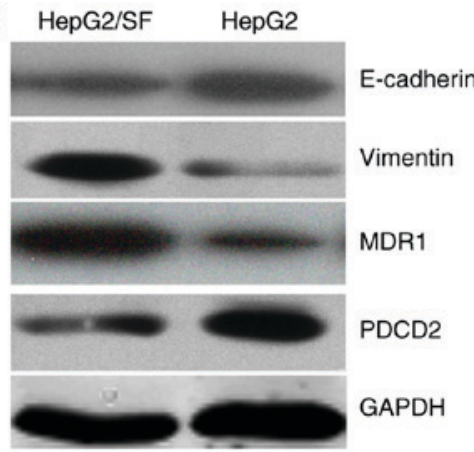

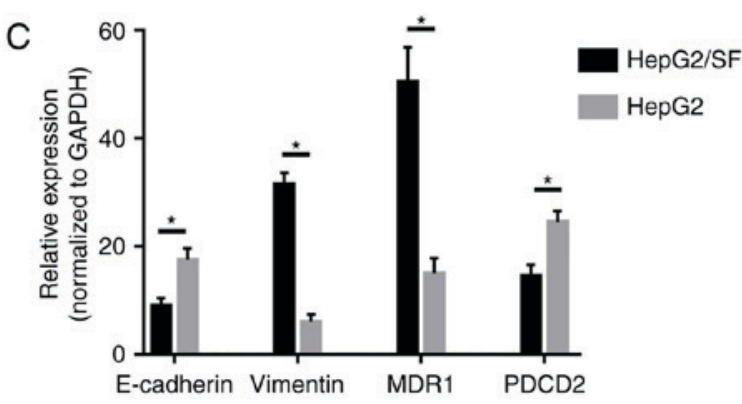

Figure 1. Decreased expression of PDCD2 accompanies MDR and epithelial-mesenchymal transition phenotypes in the HepG2/SF cell line. (A) HepG2/SF exhibited elevated IC50 for sorafenib compared with its matched maternal cell line. (B) Western blot demonstrates that HepG2/SF cells exhibited higher expression levels of Vimentin and MDR1, and lower expression levels of E-cadherin and PDCD2, compared with its matched maternal cell line. (C) Quantification of protein expression. Data are presented as the mean \pm standard deviation of three independent tests. ${ }^{*} \mathrm{P}<0.05,{ }^{* * * *} \mathrm{P}<0.001$. HepG2/SF, sorafenib-resistant HepG2 cells; PDCD2, programmed cell death domain 2; MDR, multidrug resistance; IC50, half maximal inhibitory concentration.

Statistical analysis. GraphPad Prism 7.0 (GraphPad Software, Inc.) was used to perform data analysis. All data are presented as the mean \pm standard deviation of at least three independent experiments. Student's t-test was used to determine significant differences between groups. $\mathrm{P}<0.05$ was considered to indicate a statistically significant difference.

\section{Results}

Sorafenib-resistant HepG2 cells exhibit EMT and MDR phenotypes, and decreased expression of PDCD2. The sorafenib-resistant HepG2 cell line (HepG2/SF) was successfully established, determined by the IC50 for sorafenib (Fig. 1A). The MDR1 level, which represents the MDR phenotype, was significantly increased in the sorafenib-resistant HepG 2 cell line compared with that in its matched sensitive cell line (Fig. 1B and C). Secondly, the expression of PDCD2 was analyzed, and the results demonstrated that the sorafenib-resistant HepG2 cells had a lower expression level of PDCD2. Furthermore, the expression of epithelial marker E-cadherin was decreased and that of the mesenchymal marker Vimentin was increased (Fig. 1B and C). The reduced expression of PDCD2 and the elevated EMT and MDR in the sorafenib-resistant HepG2 cell line preliminarily indicated that PDCD2 may be involved in the formation of MDR by regulating EMT.

PDCD2 reverses the drug resistance of sorafenib-resistant HepG2 cells. To further examine the function of PDCD2 in the process of MDR, the IC50 was detected following transfection of the sorafenib-resistant HepG2 cells with PDCD2. The results showed that the overexpression of PDCD2 significantly increased the sorafenib-induced cytotoxicity (Fig. 2A). Furthermore, the expression of MDR1 was decreased following transfection with PDCD2 (Fig. 2B and C). The results preliminarily demonstrated that PDCD2 may reverse the MDR of sorafenib-resistant liver cancer cells.

PDCD2 facilitates cell apoptosis and suppresses cell migration in sorafenib-resistant HepG2 cells. To further detect the function of PDCD2 involved in MDR, the apoptotic rates of sorafenib-resistant HepG2 cells transfected with PDCD2 were measured following treatment with sorafenib. As expected, the overexpression of PDCD2 enhanced sorafenib-induced apoptosis in the drug-resistant cells as revealed by flow cytometry (Fig. 3A). Furthermore, PDCD2 reduced the migration ability of sorafenib-resistant HepG2 cells (Fig. 3B). Consistent with the proposed function of PDCD2, the results of the western blotting demonstrated that PDCD2 upregulated the apoptotic-associated proteins and downregulated migration-associated proteins (Fig. 3C).

PDCD2 reduces EMT and MDR phenotypes in a Snail-dependent manner in sorafenib-resistant HepG2 cells. Given the fact that PDCD2 sensitizes sorafenib-resistant HepG2 cells to sorafenib, and the higher EMT phenotype is responsible for MDR, it was hypothesized that PDCD2 may affect EMT and be involved in the process of reversing MDR. The results of the western blotting demonstrated that the expression of E-cadherin was increased whereas the expression of Vimentin was decreased following transfection of the sorafenib-resistantHepG2 cells withPDCD2.Snail,azinc-finger transcriptional repressor, is critical in EMT-mediated tumor metastasis. Its expression was additionally downregulated in 
A

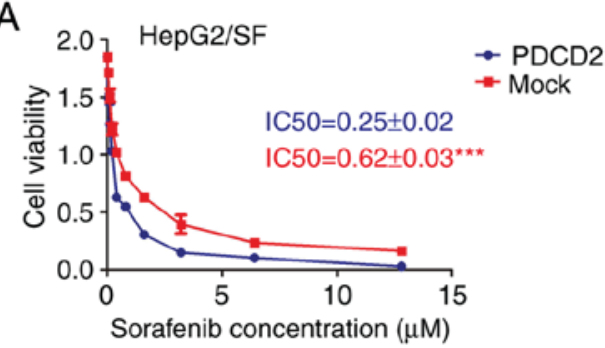

B
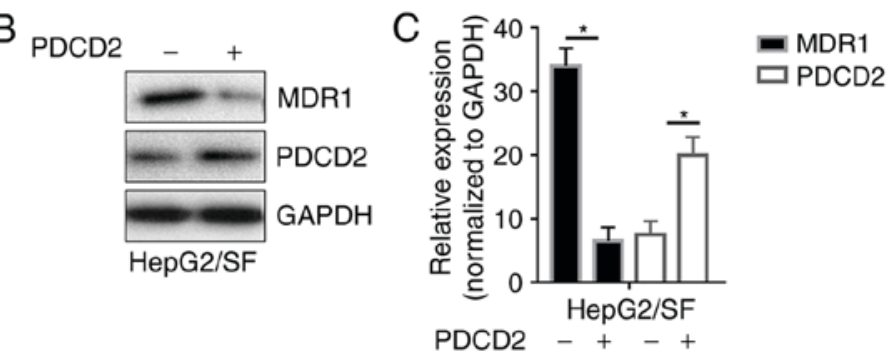

Figure 2. PDCD2 significantly decreases the MDR phenotype in the HepG2/SF cell line. (A) Overexpression of PDCD2 significantly reduced the IC50 value for sorafenib in the HepG2/SF cell line. (B) Western blot demonstrates transfection with PDCD2 decreased the expression of MDR1 in the HepG2/SF cell line. (C) Quantification of protein expression. Data are presented as the mean \pm standard deviation of three independent tests. ${ }^{*} \mathrm{P}<0.05,{ }^{* * * *} \mathrm{P}<0.001$. HepG2/SF, sorafenib-resistant HepG2 cells; PDCD2, programmed cell death domain 2; MDR, multidrug resistance; IC50, half maximal inhibitory concentration.

A

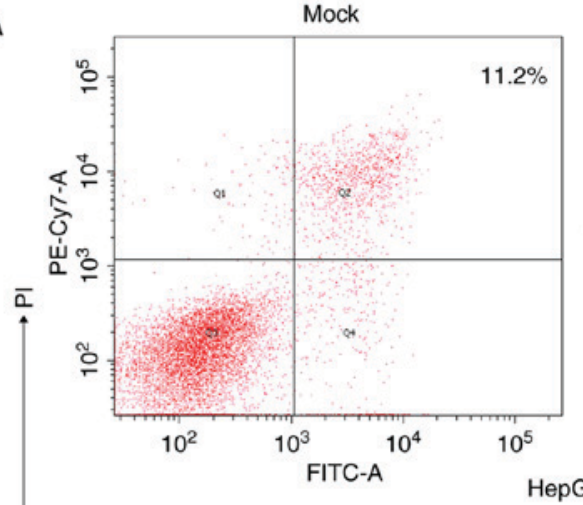

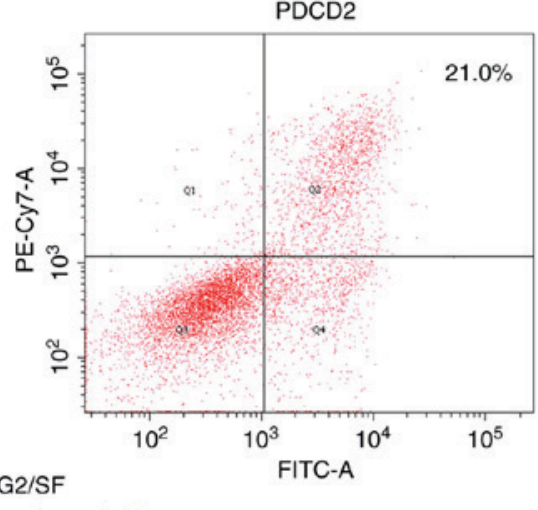

Annexin V
B

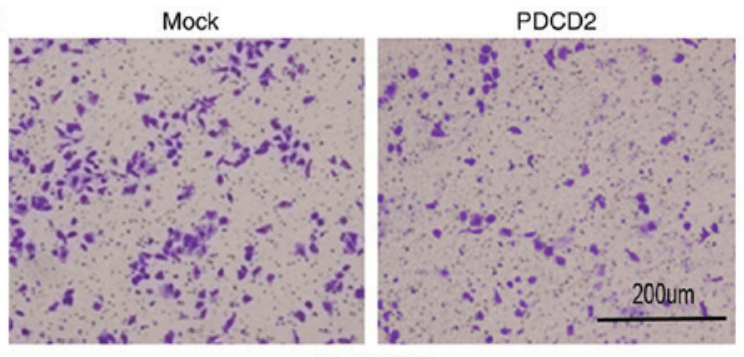

HepG2/SF

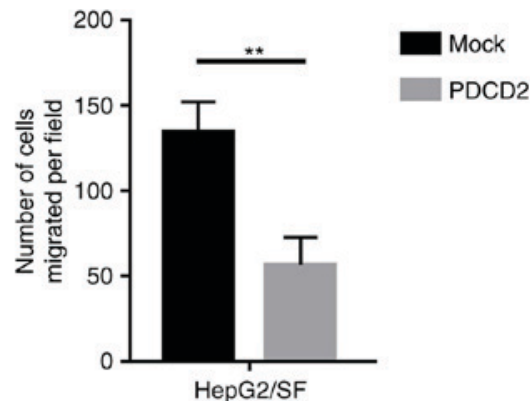

C
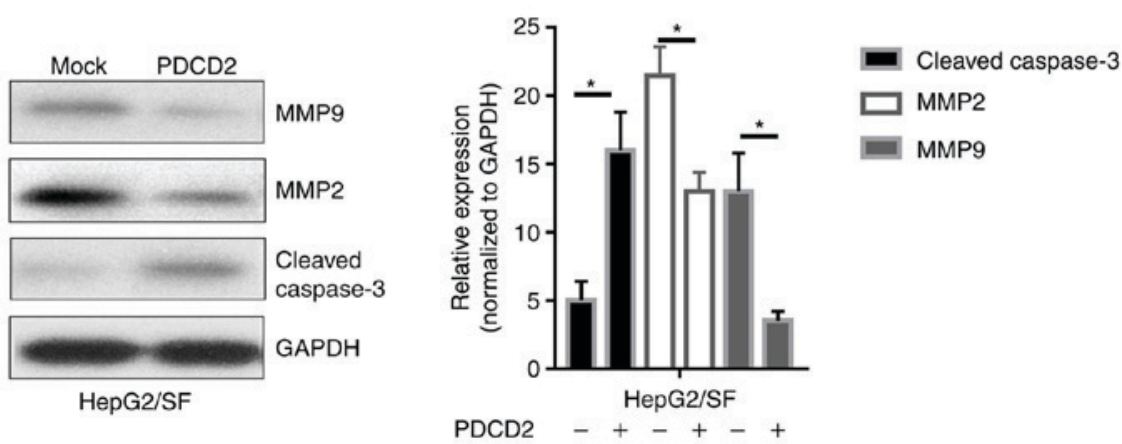

Figure 3. PDCD2 increases sorafenib-induced apoptosis and decreases cell metastatic rate in the HepG2/SF cell line. (A) Overexpression of PDCD2 enhanced sorafenib induced apoptosis, determined by flow cytometry. (B) PDCD2 decreased the metastatic rate of the HepG2/SF cell line (magnification, x20). (C) Western blotting demonstrates increased expression of cleaved caspase 3 and reduced expression of MMP2 and MMP9 following transfection with PDCD2. The data are presented as the mean \pm standard deviation of three independent tests. ${ }^{*} \mathrm{P}<0.05,{ }^{* *} \mathrm{P}<0.01$. HepG2/SF, sorafenib-resistant HepG2 cells; PDCD2, programmed cell death domain 2; MMP, matrix metalloproteinase; PI, propidium iodide; FITC, fluorescein isothiocyanate.

the sorafenib-resistant HepG2 cells transfected with PDCD2 (Fig. 4A and B). A Snail interfering vector (Snail-sh) was transfected into HepG2/SF cells, and the results demonstrated a significant downregulation of Snail expression as presented in Fig. 4C and D. Cotransfection of the HepG2/SF cells with PDCD2 and Snail-sh plasmids partially blunted the MDR and EMT phenotypes, indicating that PDCD2 likely reverses MDR and EMT in a Snail-dependent manner (Fig. 4E and F). 
A

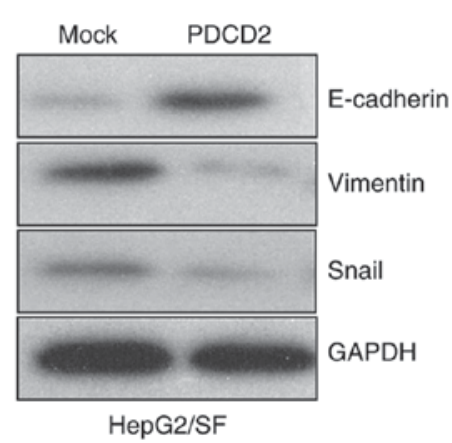

HepG2/SF
B

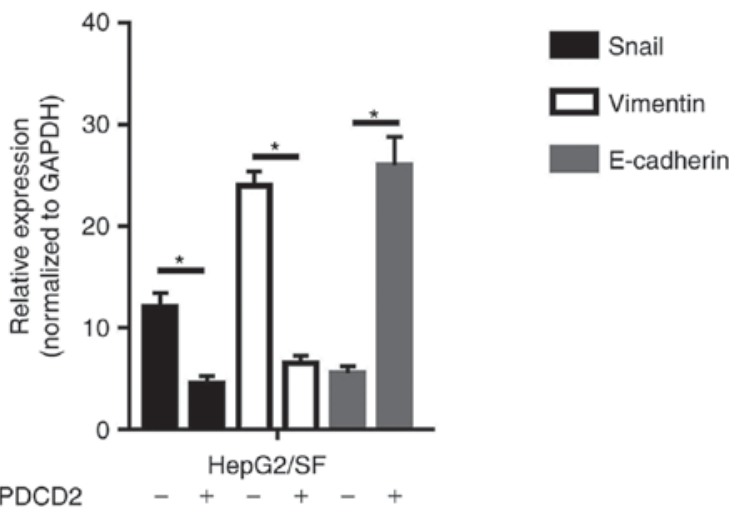

C

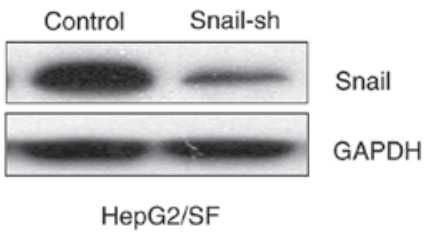

$\mathrm{E}$

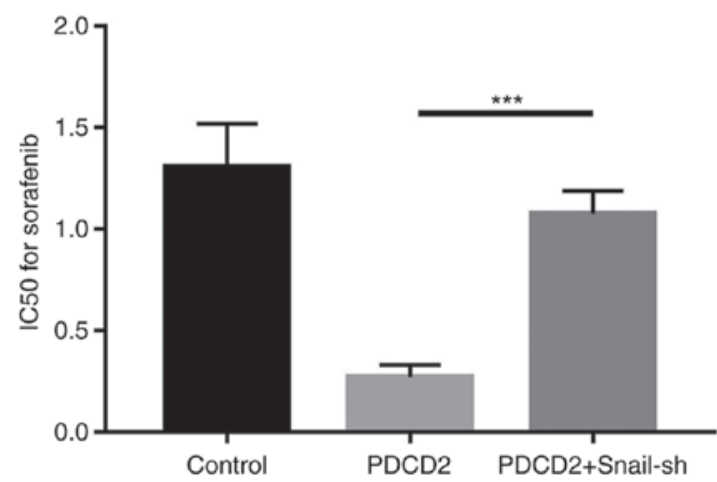

D

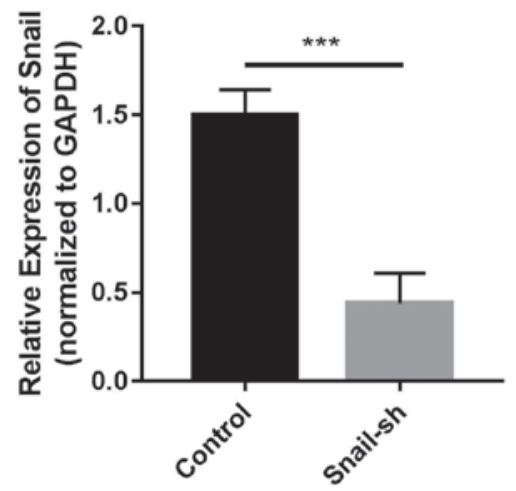

$\mathrm{F}$

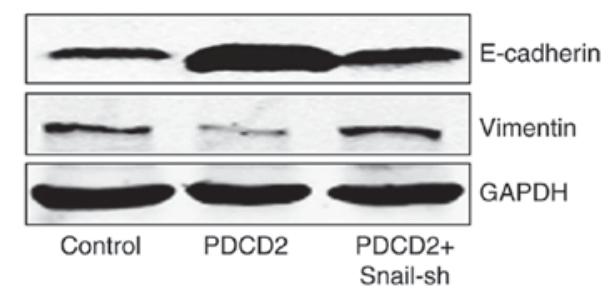

Figure 4. PDCD2 inhibits EMT and MDR phenotypes through suppression of Snail. (A) Western blotting and (B) quantification of the results demonstrated that PDCD2 decreased the expression of Vimentin and Snail and increased the expression of E-cadherin, indicating that PDCD2 may regulate EMT by the suppression of Snail. (C) Western blotting and (D) quantification of the results suggested that Snail-sh significantly decreased the expression of Snail. (E) Co-transfection with PDCD2 and Snail-sh plasmids in HepG2/SF cells blunted the function of PDCD2, indicating that PDCD2 reversed MDR in a Snail-dependent manner. (F) Co-transfection with PDCD2 and Snail-sh plasmids in HepG2/SF cells partially eliminated the EMT phenotype. Data are presented as the mean \pm standard deviation of three independent tests. ${ }^{*} \mathrm{P}<0.05,{ }^{* * *} \mathrm{P}<0.001$. HepG2/SF, sorafenib-resistant HepG2 cells; PDCD2, programmed cell death domain 2; MDR, multidrug resistance; EMT, epithelial-mesenchymal transition; IC50, half maximal inhibitory concentration; sh, small hairpin.

From these results, it was ascertained that PDCD2 controls EMT via the downregulation of Snail.

\section{Discussion}

Sorafenib, with anti-angiogenic and antiproliferative effects, is a multi-kinase inhibitor that suppresses the MAPK/extracellular signal-regulated kinase, vascular endothelial growth factor receptor and platelet-derived growth factor receptor signaling pathways (15). Generally, treatment with sorafenib leads to tumor angiogenesis suppression, cell cycle arrest and elevated apoptosis $(19,20)$. However, resistance to sorafenib is a principal cause of antineoplastic treatment failure, particularly in certain patients with advanced liver cancer under long-term treatment, which causes oncogenic relapse or distant metastasis (21). Accumulating evidence has demonstrated that EMT is the principal cause of sorafenib resistance in liver cancer cells $(10,22)$. In the present study, a sorafenib-resistant HepG2 cell line was established from long-term exposure to high-dose sorafenib. Therefore, the sorafenib-resistant HepG2 cells exhibited MDR, EMT phenotypes and decreased expression of PDCD2, preliminarily indicating that PDCD2 may be involved in the process of drug resistance by modulating EMT. 
To further investigate the function of PDCD2 in sorafenib-resistant HepG2 cell lines in the present study, the IC50 value for sorafenib and the expression levels of MDR1 following transfection with PDCD2 were detected. The results suggested that the overexpression of PDCD2 enhanced the inhibitory effect of sorafenib, as determined by the decreased IC50 value and decreased expression of MDR1. Notably, the overexpression of PDCD2 significantly increased sorafenib-induced cytotoxicity and apoptosis, and decreased the migration rate, demonstrating that PDCD2 may reverse the MDR and EMT phenotypes in sorafenib-resistant HepG2 cells. Generally, EMT is a normal developmental program that promotes cancer cells to trigger abnormal cell migration, invasion and drug resistance $(12,23)$. Therefore, EMT, as a critical regulator, is closely associated with the CSC phenotype and is a prerequisite for metastasis. The induction of EMT in epithelial cells leads to CSC characteristics, including increased stem-cell marker expression, enhanced ability to metastasize and drug resistance (24). A number of previous studies have identified the association between EMT and drug resistance (10-12,25). In the present study, the increased expression of mesenchymal markers, including vimentin, and the inhibition of epithelial markers, including E-cadherin, were observed in the sorafenib-resistant liver cancer cell lines. Mechanistically, octamer binding transcription factor 4 and Nanog are essential for the maintenance of the stem cell phenotype that hijacks liver cancer cells with CSC and EMT phenotypes via activation of the signal transducer and activator of transcription 3/Snail pathway (26). Oncogenes, including epidermal growth factor receptor, Akt and nuclear factor- $\kappa \mathrm{B}$ additionally contribute to EMT (27). However, the molecular mechanisms of how PDCD2 influences sorafenib resistance by regulating EMT in cancer cells require further elucidation.

Numerous mechanisms involved in MDR are important in the drug resistance of liver cancer, including the drug efflux pump (e.g. MDR1), EMT and DNA damage repair (28-30). EMT is more associated with the acquisition of the MDR phenotypes in liver cancer. For example, liver cancer cells with MDR have been shown to exhibit enhanced metastatic activity, and upregulated expression of $\mathrm{N}$-cadherin and Vimentin in a calcium-dependent manner (31). In addition, a previous study demonstrated that liver cancer cells underwent EMT and exhibited increased invasiveness and MDR phenotypes when exposed to hypoxia (32). A number of transcriptional repressors, including the Snail/Slug family, function as a molecular switch of EMT (33). The present study examined the crosstalk between EMT and MDR involved in the acquired drug resistance to sorafenib in liver cancer, and demonstrated that the expression of Snail was decreased when the cells overexpressed PDCD2. The Snail transcription factor is pivotal in the expression of mesenchymal markers, including Vimentin, MMP2 and MMP9 in liver cancer cells (34). Mechanistically, Snail is involved in EMT via the downregulation of cell metastasis by binding several E-boxes located in the E-cadherin promoter region (35). The overexpression of Snail facilitates the acquisition of P-glycoprotein-mediated MDR (36). Co-transfection of PDCD2 and Snail-sh plasmids into HepG2/SF cells partially blunted the MDR and EMT phenotypes, indicating that PDCD2 likely reversed MDR and EMT in a Snail-dependent manner. Therefore, the results indicated that PDCD2 modulates EMT by the suppression of Snail in drug-resistant liver cancer cells.

In conclusion, the present study demonstrated that sorafenib-resistant HepG2 cells exhibitEMT, MDR phenotypes and downregulated expression of PDCD2. The overexpression of PDCD2 suppressed sorafenib-resistant HepG2 cells from undergoing EMT and metastasis, and promoted cell apoptosis. Mechanistically, PDCD2 modulated EMT by the suppression of Snail in drug-resistant HepG2 cells. The results additionally identified that PDCD2, as a pivotal regulator of EMT, may serve as a potential therapeutic target in the treatment of sorafenib-resistant liver cancer.

\section{Acknowledgements}

Not applicable.

\section{Funding}

No funding was received.

\section{Availability of data and materials}

The datasets used and/or analyzed during the present study are available from the corresponding author on reasonable request.

\section{Authors' contributions}

LG and HL designed and conducted the experiments; MW and NL contributed to the statistical analysis. The manuscript was drafted by LG. All authors read and approved the manuscript.

\section{Ethics approval and consent to participate}

Not applicable.

\section{Patient consent for publication}

Not applicable.

\section{Competing interests}

The authors declare that they have no competing interests.

\section{References}

1. Baron BW, Anastasi J, Thirman MJ, Furukawa Y, Fears S, Kim DC, Simone F, Birkenbach M, Montag A, Sadhu A, et al: The human programmed cell death-2 (PDCD2) gene is a target of BCL6 repression: Implications for a role of BCL6 in the down-regulation of apoptosis. Proc Natl Acad Sci USA 99: 2860-2865, 2002.

2. Vaux DL and Häcker G: Cloning of mouse RP-8 cDNA and its expression during apoptosis of lymphoid and myeloid cells. DNA Cell Biol 14: 189-193,1995.

3. Yang Y, Jin Y and Du W: Programmed cell death 2 functions as a tumor suppressor in osteosarcoma. Int J Clin Exp Pathol 8: 10894-10900, 2015.

4. Zhang J, Wei W, Jin HC, Ying RC, Zhu AK and Zhang FJ: Programmed cell death 2 protein induces gastric cancer cell growth arrest at the early $\mathrm{S}$ phase of the cell cycle and apoptosis in a p53-dependent manner. Oncol Rep 33: 103-110, 2015. 
5. Wang W, Song XW, Bu XM, Zhang N and Zhao CH: PDCD2 and $\mathrm{NCoR} 1$ as putative tumor suppressors in gastric gastrointestinal stromal tumors. Cell Oncol (Dordr) 39: 129-137, 2016

6. Barboza N, Minakhina S, Medina DJ, Balsara B, Greenwood S, Huzzy L, Rabson AB, Steward R and Schaar DG: PDCD2 functions in cancer cell proliferation and predicts relapsed leukemia. Cancer Biol Ther 14: 546-555, 2013.

7. Kars MD, Iseri OD and Gündüz U: A microarray based expression profiling of paclitaxel and vincristine resistant MCF-7 cells. Eur J Pharmacol 657: 4-9, 2011.

8. Forner A, Llovet JM and Bruix J: Hepatocellular carcinoma. Lancet 379: 1245-1255, 2012.

9. Rani B, Malfettone A, Dituri F, Soukupova J, Lupo L, Mancarella S, Fabregat I and Giannelli G: Galunisertib suppresses the staminal phenotype in hepatocellular carcinoma by modulating CD44 expression. Cell Death Dis 9: 373, 2018.

10. Mir N, Jayachandran A, Dhungel B, Shrestha R and Steel JC: Epithelial-to-mesenchymal transition: A mediator of sorafenib resistance in advanced hepatocellular carcinoma. Curr Cancer Drug Targets 17: 698-706, 2017.

11. Miyazaki H, Takahashi RU, Prieto-Vila M, Kawamura Y, Kondo S, Shirota T and Ochiya T: CD44 exerts a functional role during EMT induction in cisplatin-resistant head and neck cancer cells. Oncotarget 9: 10029-10041, 2018.

12. Park JH, Shin JE and Park HW: The role of hippo pathway in cancer stem cell biology. Mol Cells 41: 83-92, 2018.

13. El-Khoueiry AB, O'Donnell R, Semrad TJ, Mack P, Blanchard S, Bahary N, Jiang Y, Yen Y, Wright J, Chen $\mathrm{H}$, et al: A phase I trial of escalating doses of cixutumumab (IMC-A12) and sorafenib in the treatment of advanced hepatocellular carcinoma. Cancer Chemother Pharmacol 81: 957-963, 2018.

14. Zhang K, Chen J, Zhou H, Chen Y, Zhi Y, Zhang B, Chen L, Chu X, Wang R and Zhang C: PU.1/microRNA-142-3p targets ATG5/ATG16L1 to inactivate autophagy and sensitize hepatocellular carcinoma cells to sorafenib. Cell Death Dis 9: 312 , 2018.

15. Nagai T, Arao T, Furuta K, Sakai K, Kudo K, Kaneda H, Tamura D, Aomatsu K, Kimura H, Fujita Y, et al: Sorafenib inhibits the hepatocyte growth factor-mediated epithelial mesenchymal transition in hepatocellular carcinoma. Mol Cancer Ther 10: 169-177, 2011.

16. Dong J, Zhai B, Sun W, Hu F, Cheng H and Xu J: Activation of phosphatidylinositol 3-kinase/AKT/snail signaling pathway contributes to epithelial-mesenchymal transition-induced multi-drug resistance to sorafenib in hepatocellular carcinoma cells. PLoS One 12: e0185088, 2017.

17. van Malenstein H, Dekervel J, Verslype C, Van Cutsem E, Windmolders P, Nevens F and van Pelt J: Long-term exposure to sorafenib of liver cancer cells induces resistance with epithelial-to-mesenchymal transition, increased invasion and risk of rebound growth. Cancer Lett 329: 74-83, 2013.

18. Zhao P, Wang S, Jiang J, Liu H, Zhu X, Zhao N, Li J, Yin Y, Pan X, Yang X, et al: TIPE2 sensitizes osteosarcoma cells to cis-platin by down-regulating MDR1 via the TAK1-NF- $\mathrm{KB}$ and -AP-1 pathways. Mol Immunol 101: 471-478, 2018.

19. Marra M, Sordelli IM, Lombardi A, Lamberti M, Tarantino L, Giudice A, Stiuso P, Abbruzzese A, Sperlongano R, Accardo M, et al: Molecular targets and oxidative stress biomarkers in hepatocellular carcinoma: An overview. J Transl Med 9: 171, 2011.

20. Zhao P, Li M, Wang Y, Chen Y, He C, Zhang X, Yang T, Lu Y, You J, Lee RJ and Xiang G: Enhancing anti-tumor efficiency in hepatocellular carcinoma through the autophagy inhibition by miR-375/sorafenib in lipid-coated calcium carbonate nanoparticles. Acta Biomater 72: 248-255, 2018.
21. Caraglia M, Giuberti G, Marra M, Addeo R, Montella L, Murolo M, Sperlongano P, Vincenzi B, Naviglio S, Prete SD, et al: Oxidative stress and ERK1/2 phosphorylation as predictors of outcome in hepatocellular carcinoma patients treated with sorafenib plus octreotide LAR. Cell Death Dis 2: e150, 2011.

22. Chen J, Jin R, Zhao J, Liu J, Ying H, Yan H, Zhou S, Liang Y, Huang D, Liang X, et al: Potential molecular, cellular and microenvironmental mechanism of sorafenib resistance in hepatocellular carcinoma. Cancer Lett 367: 1-11, 2015.

23. Shibue T and Weinberg RA: EMT, CSCs, and drug resistance: The mechanistic link and clinical implications. Nat Rev Clin Oncol 14: 611-629, 2017.

24. Otsuki Y, Saya H and Arima Y: Prospects for new lung cancer treatments that target EMT signaling. Dev Dyn 247: 462-472, 2018.

25. Zhou L, Lv X, Yang J, Zhu Y, Wang Z and Xu T: Overexpression of Napsin A resensitizes drug-resistant lung cancer A549 cells to gefitinib by inhibiting EMT. Oncol Lett 16: 2533-2538, 2018.

26. Yin X, Zhang BH, Zheng SS, Gao DM, Qiu SJ, Wu WZ and Ren ZG: Coexpression of gene Oct4 and Nanog initiates stem cell characteristics in hepatocellular carcinoma and promotes epithelial-mesenchymal transition through activation of Stat3/Snail signaling. J Hematol Oncol 8: 23, 2015.

27. Zheng HC: The molecular mechanisms of chemoresistance in cancers. Oncotarget 8: 59950-59964, 2017.

28. Teicher BA: Acute and chronic in vivo therapeutic resistance Biochem Pharmacol 77: 1665-1673, 2009.

29. van Zijl F, Zulehner G, Petz M, Schneller D, Kornauth C, Hau M, Machat G, Grubinger M, Huber H and Mikulits W: Epithelial-mesenchymal transition in hepatocellular carcinoma. Future Oncol 5: 1169-1179, 2009.

30. Zhang H, Mizumachi T, Carcel-Trullols J, Li L, Naito A, Spencer HJ, Spring PM, Smoller BR, Watson AJ, Margison GP, et al: Targeting human 8-oxoguanine DNA glycosylase (hOGG1) to mitochondria enhances cisplatin cytotoxicity in hepatoma cells. Carcinogenesis 28: 1629-1637, 2007.

31. Wen L, Liang C, Chen E, Chen W, Liang F, Zhi X, Wei T, Xue F, Li G, Yang Q, et al: Regulation of Multi-drug Resistance in hepatocellular carcinoma cells is TRPC6/Calcium dependent. Sci Rep 6: 23269, 2016.

32. Jiao M and Nan KJ: Activation of PI3 kinase/Akt/HIF-1a pathway contributes to hypoxia-induced epithelial-mesenchymal transition and chemoresistance in hepatocellular carcinoma. Int J Oncol 40: 461-468, 2012.

33. Peinado H, Olmeda D and Cano A: Snail, Zeb and bHLH factors in tumour progression: An alliance against the epithelial phenotype? Nat Rev Cancer 7: 415-428, 2007.

34. Chen JS, Li HS, Huang JQ, Zhang LJ, Chen XL, Wang Q, Lei J, Feng JT, Liu Q and Huang XH: Down-regulation of Gli-1 inhibits hepatocellular carcinoma cell migration and invasion. Mol Cell Biochem 393: 283-291, 2014

35. Zucchini-Pascal N, Peyre L and Rahmani R: Crosstalk between beta-catenin and snail in the induction of epithelial to mesenchymal transition in hepatocarcinoma: Role of the ERK1/2 pathway. Int J Mol Sci 14: 20768-20792, 2013.

36. Li W, Liu C, Tang Y, Li H, Zhou F and Lv S: Overexpression of Snail accelerates adriamycin induction of multidrug resistance in breast cancer cells. Asian Pac J Cancer Prev 12: 2575-2580, 2011.

This work is licensed under a Creative Commons

Attribution-NonCommercial-NoDerivatives 4.0 International (CC BY-NC-ND 4.0) License. 